Community + Culture features practitioner perspectives on designing technologies for and with communities. We highlight compelling projects and provocative points of view that speak to both community technology practice and the interaction design field as a whole. - Sheena Erete, Editor

\title{
Using Black Feminist Epistemologies and Activist Frameworks to Counter Structural Racism in Design
}

Sheena Erete, DePaul University

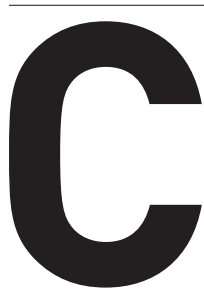

ommunity and Culture

has always been one of my favorite forums in Interactions. Since becoming a contributing editor two years ago, I have intentionally not written an article for the forum, instead choosing to elevate voices in the field who typically may not have been heard. I am proud of the topics we have covered, including intersectionality, gender inclusivity in surveys, bias and ethics in automation, and monolingualism. However, given that this issue of Interactions focuses specifically on racial equity, I am compelled to share my thoughts on how racial inequity impacts every aspect of design, design research, and research partnerships. Like my grandmother always said, "Say something when something needs to be said.” So I will.

In the past year, the murders of Black people by police in the U.S. has led to renewed interest in examining and addressing structural racism and oppression, both in society at large and in the fields of computing and design. Like many Black people who live in the U.S., I find incidents such as the murders of George Floyd, Breonna Taylor, Alton Sterling, and others neither surprising nor shocking. What has been shocking to me is the number of senior scholars in computing and design who study issues of equity who are shocked and dismayed that racism still exists, and that they seem unaware that the lived experiences of Black people differ from their own. From a more global perspective, some have claimed that racism exists only in the U.S., attempting to exempt other countries from being called out for their unfair treatment of Black people in the diaspora and absolve these countries of their contributions to colonization, imperialism, and slavery. There is both quantitative (i.e., statistics if that's your epistemic orientation) and qualitative (i.e., published academic articles as well as the lived experiences of most Black people you know) evidence of the outcomes of structural racism. This is not to say that individual exceptionalism does not exist. Instead, these outcomes suggest that structures are in place that impede the liberties and freedoms of Black people, making it challenging for them to navigate systems that are rooted in the oppression of people who are not white, male, cisgender, able-bodied, Western, and so on. These structures are embedded in both society and design, as several scholars have recently brought

Insights

$\rightarrow$ We can move toward countering structural racism and oppression in design by using frameworks and approaches that decenter whiteness, such as Black feminist thought, intersectionality, and transformative justice.

$\rightarrow$ Nothing about us without us! The powerful mantra from the disability community is a model for achieving racial equity in design research, suggesting that research about Black people should be in partnership with Black scholars and using frameworks that center Black people and culture. to light $[1,2,3,4]$.

After working for over a decade on issues that are a direct outcome of structural racism and oppressionviolence, health inequity, lack of education opportunities, political (dis)engagement - I believe that as designers and researchers, we must: 1) move beyond technology innovation toward addressing structural change by wrestling with issues of power that are rooted in historical and current policies and practices that perpetuate oppression; and 2) examine our role in organizations, institutions, fields, and societies that have engaged in the oppression of nonwhite people, whether it be slavery, colonization, unfair labor practices, or others. I, along with my amazing collaborators, have thought long about issues of structural racism and oppression, leveraging Black feminist epistemologies such as Black feminist thought and intersectionality as well as liberatory, activist approaches such as transformative justice both to examine these histories and powers and to design technologies, policies, and practicies that counter and resist systemic oppression and harm. In this article, I briefly describe how we use these frameworks in our research and design praxis, positing that without critically examining histories of power in society and in our design and research methods, we will never come close to achieving racial equity.

\section{BLACK FEMINIST THOUGHT AND INTERSECTIONALITY}

Introduced by Patricia Hill Collins and rooted in social justice and activism, Black feminist thought 
(BFT) is a critical social theory that positions and values Black women's lived experiences as knowledge to understand structural oppression and injustice by interrogating systems of power [5]. Collins writes, "Whether viewed through the lens of a single system of power, or through that of intersecting oppression, any particular matrix of domination is organized via four interrelated domains of power, namely, the structural, disciplinary, hegemonic [now called cultural] and interpersonal domains. Each serves a particular purpose. The structural domain organizes oppression, whereas the disciplinary domain manages it. The [cultural] domain justifies oppression while the interpersonal domain influences everyday lived experience and the individual consciousness that ensues" [5].

Based on the work of Black, Latina, Indigenous, and Native women dating back to the 19th century, intersectionality is a critical framework that examines the ways in which systemic oppression shifts and changes in interaction with multiple, overlapping, and intersecting elements of people's identities [6]. Intersectionality posits that inequality is best understood by exploring the intersection of identities (race, gender, class, ability, nation, etc.) rather than by looking at them in isolation, and that those who have been historically oppressed engage in acts of resistance as a form of social justice in an effort to create a more equitable society [6]. With a false sense of neutrality and colorblindness, the computing and design fields tend to ignore the fact that most of our practices (e.g., research frameworks, data collection and analysis methods), partnership strategies, funding mechanisms, and dissemination strategies are created from intersecting systems of power that attend to and center whiteness, capitalism, individualism, and innovation.

Leveraging these two frameworks, my colleagues Yolanda Rankin, Jakita Thomas, and I have focused on understanding the lived experiences of Black women in computing and HCI and the violence that they experience $[1,4]$. Our work suggests that far from being equitable and colorblind, as it

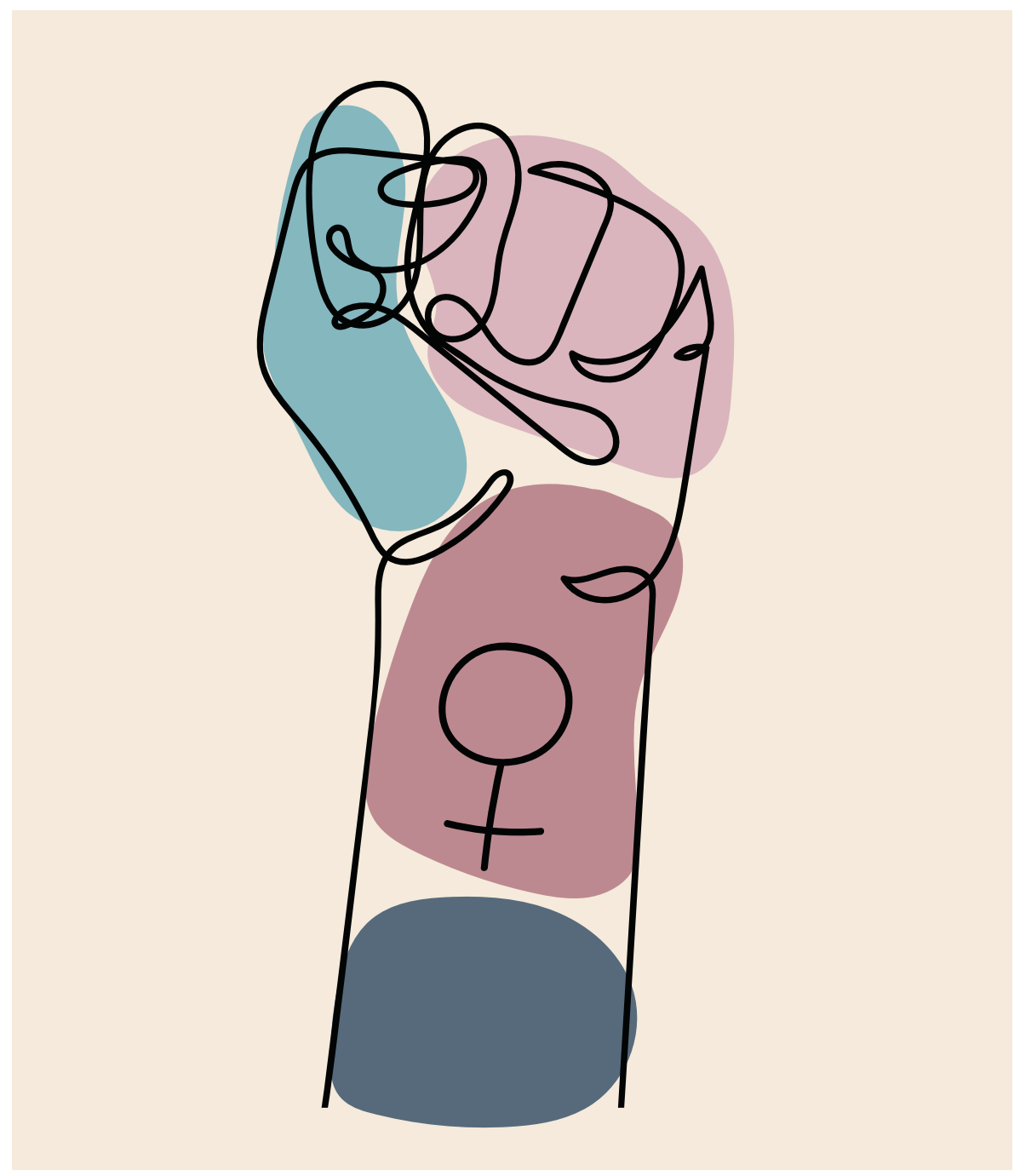

attempts to present itself, the field instead causes harm and trauma to its Black women members. Solutions to addressing the structures that cause these harms include, but are not limited to, transforming the education experience, the peer review process, and who is invited to serve in leadership positions [1].

In our recent work [7], Rankin, Thomas, and I introduce the intersectional analysis of power as a method to analyze systems of power in computing, design, and HCI by reflecting on historical and current interconnected systems of power and

Far from being equitable and colorblind, the field instead causes harm and trauma to its Black women members. oppression and how they work together to create and maintain inequitable social outcomes in design, design research, and research partnerships. To illustrate, we dissected power in the design and research process using the context of co-designing a mobile application with street outreach workers to support their communityled violence-prevention efforts [8]. We examined both the criminality of Black bodies and the deficit narratives of Black communities while also interrogating our role as researchers working with these communities. We found that, as Black feminists, we naturally act in resistance to methods that are rooted in whiteness and domination. In our initial engagement, for example, the community organization led the project framing and the research questions were co-designed in equitable ways such that they benefit the community organization and the researcher's goals. We thought of how grant funding 


\section{DFORUM COMMUNITY + CULTURE}

is typically distributed (e.g., to the researcher's academic institution) and asked, What would happen if there was equity in the distribution of grant funding, where some of the grants went directly to the community partners and we were subawardees instead of the other way around? Taking this approach completely changed the nature of the research partnership. We also resisted the notion of individual exceptionalism and paternalism (concepts both rooted in white supremacy) by intentionally giving the intellectual property of the co-designed mobile application (legally as well as the code/servers) to the community partners. Understanding the intersecting systems of power, we decided to engage in shared decisionmaking with regard to the design methods used and the dissemination of the findings. Based on our self-reflection, we believe that we could have done a better job advocating for funding that allowed community partners to engage fulltime in the data-analysis process, rather than us researchers leading the data analysis and bringing our results back to the community for feedback. We didn't just reflect on these things; we pledged to take a different approach to doing data analysis in the future. This level of reflexivity is necessary for invoking structural changes that push against the status quo.

\section{TRANSFORMATIVE JUSTICE}

Originating from the practices of Native and Indigenous peoples, transformative justice is an approach to both acknowledge and respond to harm experienced by those who have historically faced systemic oppression and state violence [9]. As have others in design who have leveraged transformative justice $[8,10,11,12]$, we apply it as a method to center people, relationships, and communities, focusing on their assets and capacities to create alternative structures that are sustainable beyond our engagement.

\section{Transformative justice is not formulaic, but rather is unique to the histories and experiences of each community.}

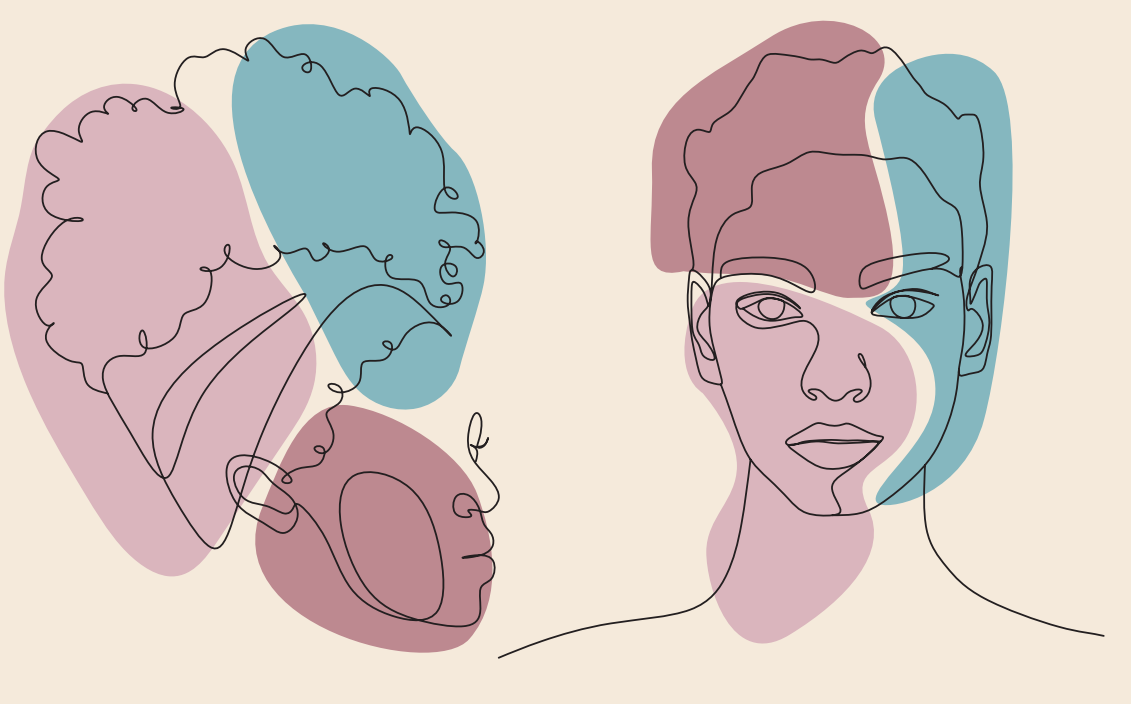

Transformative justice is not formulaic, but rather is unique to the histories and experiences of each community. We apply several principles from transformative justice in our work, including "addressing historical and structural causes of harm while also ameliorating the impacts of those harms; taking a community-centered approach that fosters relationships and leverages strengths; building safe spaces with accountability mechanisms; and developing counter-structures to violent, harmful, and oppressive policies and institutions” [12]. These transformative justice principles have a substantial impact on both the communities we serve and our development as researcher-activists [13].

As an example, in 2013 we launched Digital Youth Divas (DYD), an out-ofschool-time learning program codesigned with and for Black and Latina middle school girls to engage in STEM. As with many other NSF-sponsored projects, our goal was to encourage more diverse participation in STEM careers. By all academic measures, we have been successful: More than 500 girls have participated since its inception and DYD participants' STEM skills, confidence, and identity have increased. However, why should we use academic standards to measure success when we know that lack of STEM participation is rooted in structural racism and violence [4]? Taking a researcher-activist stance [13] toward transformation, we acknowledge the historical harms faced by the communities in which we live and work: lack of familial wealth and concentrated poverty caused by segregationist housing policies and lack of investment in Black communities; lack of access to informal learning opportunities due to school closures in Black neighborhoods and the subsequent bussing of Black youth, which takes away time that could be spent in afterschool programs; school structures that adultify and sexualize Black and Latina girls and suspend them more often than white girls for the same behavior; harmful narratives and stereotypes of who does and who does not belong in STEM; among others.

Recognizing the harms allows us to move our research and praxis beyond our initial appraoch, which consisted of culturally relevant pedagogy, a near-peer mentoring model, and a digital learning platform that fosters peer social relationships and narrative-driven projects. Instead, we moved toward addressing oppressive structures, while also acknowledging that we, as educators, researchers, computing professionals, designers, and academics, are complicit in continuing harm as members of institutions that inflict these harms. As acts of resistance, we actively attempted to create counter structures in the following ways:

- Co-selecting with community residents the physical location where DYD is held to counter harm caused by school closures

- Creating a parent and caring-adult network to help Black and Latinx families with parent-support roles when engaging in local STEM opportunities 
to build social relationships that counter the division caused by inequity in bussing

- Ensuring that our research team has a community voice through our fulltime community engagement manager, a Black woman who is a parent that lives in the community, and a steering committee that consists of parents and caring adults to help shape decisionmaking for the project

- Co-designing narratives (written books, audio stories, and short animations) that launch the STEM projects to counter hegemonic stereotypes about Black and Latina girls

- Creating social safe spaces where girls can openly talk to each other and near-peer mentors about their lived experiences

- Creating a mentor network embedded in local city funding that allows the program to be sustainable beyond grant funding to counter lack of capacity building

- Creating a collective of local STEM program providers that work together to intentionally identify harmful practices within STEM and computing education in formal and informal learning spaces.

Though the outcomes as a result of these counter structures are generally positive [12], diversity numbers in STEM and CS fields will not change until the toxic culture changes. It is therefore an act of resistance for a Black or Latina girl to refuse to sacrifice their mental and physical health and well-being to participate in STEM when the structures in placeincluding the people in power-refuse to change. Given the empty promises of diversity and inclusion initiatives, our goal now is to simply create local learning ecosystems that expose the girls to STEM environments devoid of harm and trauma that confirm that they are capable of engaging in STEM. Whether they decide to do so is up to them.

To address racial inequity in design, we must move toward designing counter structures of systems of power that were designed to lift some and oppress others. History suggests that oppression is embedded into society, and race has been constructed as a mechanism to justify the oppression of nonwhite people. Leveraging frameworks such as BFT, intersectionality, and transformative justice is one way that we can recognize the harms caused by structural racism, reflect on how we are complicit in continuing those harms, and identify ways in which we can begin to attempt to address and counter such harms.

\section{ACKNOWLEDGMENTS}

Addressing racism and structural change cannot happen without pushing against notions of individualism toward transformation through collaborative efforts. Thus, throughout this article I have used the term we, which refers to my community partners and collaborators (faculty and students) who are too numerous to name in entirety but here's a few: Natasha Smith-Walker, Jalon Arthur, Angelica Bianca, Jessa Dickinson, Alejandra Lopez Gonzalez, Yolanda Rankin, Jakita Thomas, Nichole Pinkard, Denise Nacu, Caitlin Martin, and those from the Technology for Social Good lab at DePaul and the Digital Youth Network at Northwestern University. I also thank the activist movements, community organizations, and researcher-activists who have been doing this work for years and funders for their support. Last, I am limited in my ability to cite all the related work in this article so I point to our longer academic articles for more robust reference of prior literature.

\section{ENDNOTES}

1. Erete, S., Rankin, Y.A., and Thomas, J.O. I can't breathe: Reflections from Black women in CSCW and HCI. Proc. ACM Hum.-Comput. Interact. 4, CSCW3 (Dec. 2020), Article 234; https://doi-org. ezproxy.depaul.edu/10.1145/3432933

2. Ogbonnaya-Ogburu, I.F., Smith, A.D.R., To, A., and Toyama, K. Critical race theory for HCI. Proc. of the 2020 CHI Conference on Human Factors in Computing Systems. ACM, New York, 2020, 1-16; https://doi-org.ezproxy. depaul.edu/10.1145/3313831.3376392

3. Pierre, J., Crooks, R., Currie, M., Paris, B., and Pasquetto, I. Getting ourselves together: Data-centered participatory design research \& epistemic burden. Proc. of the 2021 CHI Conference on Human Factors in Computing Systems. ACM, New York, 2021, Article 406, 1-11; https://doi-org.ezproxy.depaul. $\mathrm{edu} / 10.1145 / 3411764.3445103$
4. Rankin, Y.A., Thomas, J.O., and Erete, S. Real talk: Saturated sites of violence in CS education. Proc. of the 52nd ACM Technical Symposium on Computer Science Education. ACM, New York, 2021, 802808; https://doi-org.ezproxy.depaul. $\mathrm{edu} / 10.1145 / 3408877.3432432$

5. Collins, P.H. Black Feminist Thought. Routledge, 2000.

6. Collins, P.H. Intersectionality as Critical Social Theory. Duke Univ. Press, 2019.

7. Erete, S., Rankin, Y.A., and Thomas, J.O. A method to the madness: Applying an intersectional analysis of structural oppression and power in HCI and design. (under review).

8. Dickinson, J., Arthur, J., Shiparski, M., Bianca, A., Gonzalez, A., and Erete, S. Amplifying Community-led Violence Prevention as a Counter to Structural Oppression. Proc. ACM Hum.-Comput. Interact. 5, CSCW1 (Apr. 2021), Article 180; https://doi-org.ezproxy.depaul. $\mathrm{edu} / 10.1145 / 3449279$

9. State violence is the "physical, emotional, financial, psychological, and mental harms imposed on individuals and communities through the use of force, intimidation, or structural policies that impede individuals' and communities' freedom to thrive and grow" [12].

10. Asad., M. Prefigurative design as a method for research justice. Proc. ACM Hum.-Comput. Interact. 3, CSCW (Nov. 2019), Article 200; https://doi-org. ezproxy.depaul.edu/10.1145/3359302

11. Costanza-Chock, S. Design Fustice: Community-Led Practices to Build the Worlds We Need. MIT Press, 2020.

12. Erete, S., Thomas, K., Nacu, D., Thompson, N., Dickinson, J., and Pinkard, N. Applying a transformative justice approach to encourage the participation of Black and Latina Girls in computing. ACM Trans. Comput. Educ. (in press).

13. de Castro Leal, D., Strohmayer, A., and Krüger, M. On activism and academia: Reflecting together and sharing experiences among critical friends. Proc. of the 2021 CHI Conference on Human Factors in Computing Systems. ACM, New York, 2021, Article 303, 1-18; https://doi-org.ezproxy.depaul. edu/10.1145/3411764.3445263

(1) Sheena Erete is an associate professor in the College of Computing and Digital Media at DePaul University in Chicago, IL. Her work focuses on co-designing sustainable technologies, practices, and policies with community organizations to counter structural oppression using equity-centered, justiceoriented, assets-based approaches to research and design

$\rightarrow$ seretedacdm.depaul.edu 